\title{
Pourous nanoparticles formation using a dendrimer template
}

\author{
L. Bonaccorsi ${ }^{\mathrm{a}}$, D. Lombardo ${ }^{\mathrm{b}, *}$, A. Longo ${ }^{\mathrm{c}}$, E. Proverbio $^{\mathrm{a}}$ and P. Calandra ${ }^{\mathrm{b}}$ \\ ${ }^{a}$ Dipartimento di Chimica Industriale e Ingegneria dei Materiali, Universita Messina, S. Agata \\ (Messina), Italy \\ ${ }^{\mathrm{b}}$ CNR-IPCF, Istituto per i Processi Chimico Fisici - Sezione de Messina, Messina, Italy \\ ${ }^{\mathrm{c}}$ CNR-ISMN, Istituto per lo Studio dei Materiali Nanostrutturati - Sezione de Palermo, Palermo, Italy
}

\begin{abstract}
We describe the synthesis of porous spherical nanoaggregates derived by the controlled growth of zeolite LTA on a poliamidoamine (Pamam) dendrimer acting as nano-template. The presence of a strong condensed cationic $\mathrm{Na}^{+}$charge all around the dendrimers, which is responsible for the intense electrostatic inter-particle interaction potential, stimulate the condensed growth of the zeolitic phase onto the dendrimer substrate. Moreover, the screening produced by the zeolite grown on the dendrimer surface promote the entanglement process between the primary units with the formation of large porous clusters. The main features of the self-assembly process has been characterised by means of different techniques such as Small Angle X-ray Scattering (SAXS), Scanning Electron Microscopy (SEM), X-ray Diffraction (XRD) and Energy Dispersive X-ray (EDX) microprobe spectroscopy. The finding of our results may open new perspectives in the synthesis of organic-inorganic nanostructured materials based on mesoporous frameworks with new characteristics and properties.
\end{abstract}

Keywords: Nanostructures, zeolites, dendrimer, self-assembly

\section{Introduction}

Recently there has been increasing interest in the synthesis, characterization and processing of novel materials with controlled structural characteristics [20,30]. Particularly interesting in this respect is the construction of supra-molecular hybrid organic-inorganic nanostructures based on microporous and nanoporous materials $[1,8,17,18,31]$. Hybrid inorganic-organic nanoporous materials can be synthesized by several traditional methods including solvent-diffusion method, solvothermal synthesis (using an organic solvent), hydrothermal synthesis (using water as solvent) [28]. On the other hand the development of alternative protocols for the organic templating growth of inorganic materials represents an emerging reality due also for the interest in the fabrication of new types of porous hybrid materials with peculiar structure and functions [2,7]. These approaches, which are based on the use of a specific macromolecular template, consists in the controlled spatial growth of nanosized macromolecular assemblies which result in the formation of defect free, higher-order structures like membranes, films, coatings [11, 34]. Such self-assembly recently attracted a sensitive attention in material science $[9,16,19,25,26,36]$ due also for the important implications in the improvement of the models and theoretical approaches describing the relavant assembly mechanism involved in material science (such as formation of colloids,

\footnotetext{
${ }^{*}$ Corresponding author: D. Lombardo, CNR - Istituto per i Processi Chimico Fisici - Sezione de Messina, C. da Papardo, Messina I-98158, Italy. Tel.: +39090 39762222; Fax: +39090 3974130; E-mail: lombardo@me.cnr.it.
} 
molecular crystals, lipid bilayers, phase-separated polymers) as well as in many processes involved in life science $[10,15,22,23,35]$.

In this paper, we describe the synthesis of spherical nanoaggregates derived by the controlled growth of zeolite LTA on a carboxyl-terminated poliamidoamine (Pamam) dendrimer. More specifically we evidence that spherical pourous nanoparticle were formed when the charged terminal groups of the dendrimer interacted with the alluminosilicate components to irreversibly anchor the zeolite formation to the dendrimer surface. The use of branched polymeric materials, such as dendrimers, as nanotemplating agents has been proven as a versatile tool in the field of nanotechnology, due to the accurate control of the size of the inorganic particles involved [12,27,32,33].

\section{Materials and methods}

Carboxil-terminated polyamidoamine (Pamam) dendrimers of generation $G=3.5\left(M_{\mathrm{w}}=\right.$ $12,420 \mathrm{~g} / \mathrm{mol}$ ) were purchased from Sigma Aldrich. The dendrimers were dispersed in deionised water, while the obtained solutions were filtered with Teflon filters (filter diameter was $D=0.02 \mu \mathrm{m}$ ). The solutions were also checked by dynamic light scattering prior to SAXS measurements to remove the presence of possible aggregates in the system.

The zeolite synthesis mixtures, prepared according the standard procedure [3,4,6], had the following molar ratio: $2.0 \mathrm{NaO}_{2}: 1 \mathrm{Al}_{2} \mathrm{O}_{3}: 1.9 \mathrm{SiO}_{2}: 65 \mathrm{H}_{2} \mathrm{O}$. All alluminosilicate reactants were preliminary mixed and then added with a water solution of generation $G=3.5$ Pamam dendrimers at the concentration of $c=1 \%$ w/w (AS/Pamam).

The Small Angle X-ray Scattering (SAXS) experiments have been performed by using a laboratory instrumentation consisting of a Philips PW X-ray generator (providing $\mathrm{Cu} \mathrm{K} \alpha$, Ni-filtered X-ray radiation of wavelength $1.5418 \AA$ ) with a Kratky-type small-angle camera in the "finite slit height geometry" equipped with step scanning motor and scintillator counter as detector. All measurements were carried out at the temperature of $T=25^{\circ} \mathrm{C}$. The scattering data were normalized with respect to transmission and were corrected by the empty cell and solvent contribution.

The Electron Microscopy was performed using a scanning electron microscope JEOL 5600LV operated at $10 \mathrm{kV}$ in low-vacuum condition. The microscope was equipped with a backscattered electron detector and an EDS electronic microprobe (SEMQuant Oxford).

\section{Results and discussion}

The self-assembly process during nanoparticles formation has been investigated by means of Small Angle X-ray Scattering (SAXS) experiments. For a system composed of nearly monodisperse particles in solution the SAXS scattering intensity $I(q)$ can be expressed as a product of the form factor $P(q)$, which contains information on the shape and dimension of the scattering particles and the structure factor $S(q)$ describing the inter-particle interaction $[13,14]$,

$$
I(q)=N(\Delta \rho)^{2} P(q) S(q),
$$

where $N$ is the number density of the particles, and $\Delta \rho=\left(\rho-\rho_{0}\right)$ is the so-called "contrast" (i.e., the difference between the scattering length density of the particle $\rho$ and that of the solvent $\rho_{0}$ ). Figure 1A show the SAXS intensity profile for the G3.5 Pamam dendrimers in water solution at concentration 

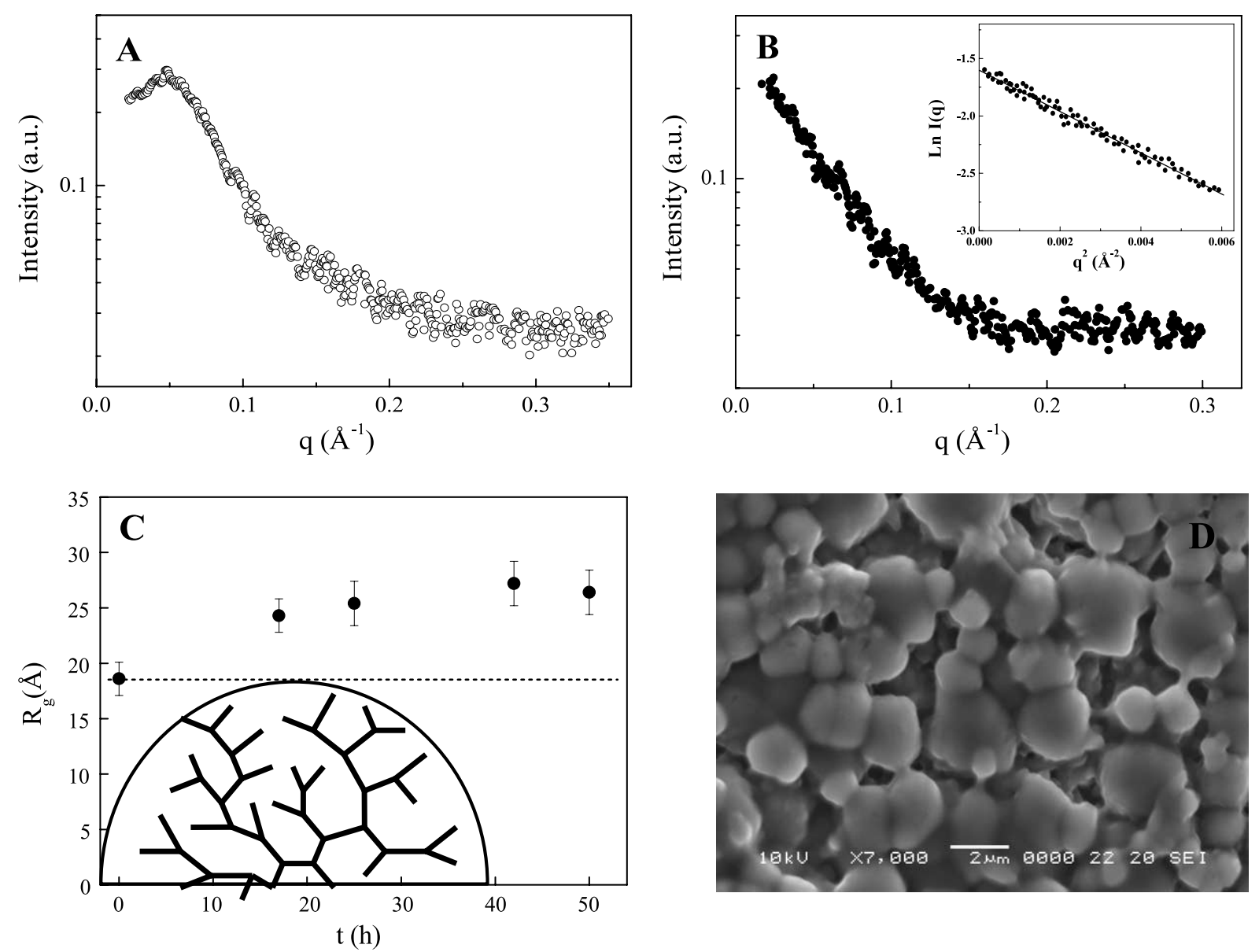

Fig. 1. (A) SAXS intensity profile of G3.5 Pamam dendrimers in water solution at the concentration of $c=1 \mathrm{wt} \%$. (B) Guinier fitting analysis of the SAXS intensity profile for the AS/Pamam system $16 \mathrm{~h}$ after the mixing of the main components. (C) Analysis of the radius of gyration $R_{\mathrm{g}}$ as a function of the elapsed time (in hours) after mixing. (D) Scanning electron microscopy images of the generated spherical supramolecular assembly for the Pamam/AS system.

$c=1 \mathrm{wt} \%$. The presence a pronounced structure factor peak, even in this dilute regime, can be traced back to the electrostatic repulsive interaction caused by the partial ionisation of the dendrimers carboxilate surface groups $[21,24,27]$.

In order to obtain direct information of the morphological features during self-assembly process all alluminosilicate reactants were preliminary mixed and then added with a water solution of generation G3.5 Pamam dendrimers at the concentration of $c=1 \% \mathrm{w} / \mathrm{w}$ (AS/Pamam). SAXS intensity profile detected $16 \mathrm{~h}$ after the mixing (Fig. 1B) show the disappearance of the structure factor peak. This indicate that the inter-particle interaction can be considered negligible (i.e., $S(q) \approx 1$ ) as a consequence of the condensed growth of the zeolitic phase onto the charged dendrimer surface. In this case the analysis of SAXS scattering intensity $I(q)$ can furnish information about the form factor $P(q)$, and the information about the dendrimer radius of gyration $R_{\mathrm{g}}$ can be obtained from the slope of the representation $\ln I(q)$ vs. $q^{2}$ in the so-called Guinier region (i.e., for $q R_{\mathrm{g}} \ll 1$ ), where the particle form factor $P(q)$ can be 
expressed as [14]:

$$
P(q)=P(0) \exp \left(-\frac{q^{2} R_{\mathrm{g}}^{2}}{3}\right) .
$$

As shown in the inset of Fig. 1B, the radii of gyration were obtained from the slope of the representation of $\ln I(q)$ vs. $q^{2}$. Results of the dimension analysis as a function of the elapsed time after the mixing is reported in Fig. 1C, which indicates that the radius of gyration $R_{\mathrm{g}}$ slightly increases with the reaction time, starting from $R_{\mathrm{g}}=18.6 \AA$ (for solely Pamam dendrimer in water solution [29]) up to $R_{\mathrm{g}}=$ $26.4 \AA$ ( $50 \mathrm{~h}$ after the mixing). This thickening of the original particles caused by the condensed growth of the zeolitic phase onto the dendrimer substrate represent the first stages of the formation process. The screening of the repulsive interaction, in fact, promote also an entanglement process between the primary units, with the formation of large clusters of the order of one micron. The formation of the supramolecular assembly probably generated by a secondary process, which has also been observed by photon correlation spectroscopy experiments [5], was finally verified by scanning electron microscopy (SEM) as shown in Fig. 1D. The back-scattered SEM image of the system, three days after the mixing, confirmed a condensation of aluminosilicate components on the large aggregates surfaces as proved by the EDX microprobe analysis.

Finally, to confirm the nature of the condensate phase, the clear synthesis solution was left reacting at room temperature for 1 week and then was dried at $40^{\circ} \mathrm{C}$ for $24 \mathrm{~h}$. The solid was collected and dried again at $80^{\circ} \mathrm{C}$ for $12 \mathrm{~h}$ and checked by XRD powder diffraction which showed the characteristic peaks of crystalline zeolite.

In conclusion, we have demonstrated the successful self-assembly of a spherical complex, driven by the incorporation of alluminosilicate in a dendrimer charged surface. More specifically we observe that spherical nanometric assemblies are formed when the carboxilate dendrimer endgroups interact with the alluminosilicate components to irreversibly anchor the zeolite formation to the dendrimer surface. Molecular driving force in this case can be traced back to the strong condensed charge $\left(\mathrm{Na}^{+}\right.$cations from the carboxylic dendrimer terminal groups and from the mother liquid of zeolite components) from the diffuse double layer in the surface of the dendrimer. This condensed charge, acting as an effective structure-directing agent casted the growth of the zeolite nanoporous structures directly on the dendrimer surface. Such materials could have numerous potential applications such as adsorption, catalysis, separation, environment protection. Moreover, they can be used to encapsulate or separate molecules depending on the sizes of molecules. The current investigations emphasize the potential of using hybrid organic-inorganic networks in the synthetic construction of functionalized materials with higher-order structures.

\section{References}

[1] P.C. Angelomé, M.C. Fuertes and G.J.A.A. Soler-Illia, Adv. Mater. 18 (2006), 2397-2402.

[2] M. Antonietti and G.A. Ozin, Chem. Eur. J. 10 (2004), $28-41$.

[3] R.M. Barrer, Hydrothermal Chemistry of Zeolites, Academic Press, London, 1982.

[4] R.M. Barrer, Synthesis of zeolites, in: Zeolites, B. Drzaj, S. Hocevar and S. Pejovnik, eds, Elsevier, Amsterdam, 1985.

[5] L. Bonaccorsi, D. Lombardo, A. Longo, E. Proverbio and A. Triolo, Macromolecules 42 (2009), 1239-1243.

[6] D. Breck, Zeolite Molecular Sieves, Structure, Chemistry and Use, Wiley, New York, 1974.

[7] C.J. Brinker, Y. Lu, A. Sellinger and H. Fan, Adv. Mater. 11 (1999), 579-585. 
[8] C.J. Brinker and G.W. Scherrer, Sol-Gel Science, The Physics and Chemistry of Sol-Gel Processing, Academic Press, San Diego, 1990.

[9] S.H. Chen, F. Malamace, A. Faraone, P. Gambadauro, D. Lombardo and W.R. Chen, Eur. Phys. J. 9 (2003), $283-286$.

[10] D. Chun, F. Wudl and A. Nelson, Macromolecules 40 (2007), 1782-1785.

[11] A.B. Descalzo, R. Martínez-Máñez, F. Sancenón, K. Hoffmann and K. Rurack, Angew. Chem. 45 (2006), 5924-5948.

[12] D. Farin and D. Avnir, Angew. Chem. Int. Ed. Engl. 30 (1991), 1379-1380.

[13] L.A. Feign and D.I. Svergun, Structure Analysis by Small-Angle X-Ray and Neutron Scattering, Plenum Press, New York, 1987.

[14] O. Glatter and O. Kratky, Small-Angle X-Ray Scattering, Academic Press, London, 1982.

[15] F. Gröhn, G. Kim, B.J. Bauer and E.J. Amis, Macromolecules 34 (2001), 2179-2185.

[16] L. He, V.M. Garamus, S.S. Funari, M. Malfois, R. Willumeit and B. Niemeyer, J. Phys. Chem. B 106 (2002), $7596-7604$.

[17] S.H. Jhung, J.-H. Lee, P.M. Forster, G. Frey, A.K. Cheetham and J.-S. Chang, Chem. Eur. J. 12 (2006), 7899-7905.

[18] P. Judenstein and C. Sanchez, J. Mater. Chem. 6 (1996), 511-525.

[19] F. Lafleche, D. Durand and T. Nicolai, Macromolecules 36 (2003), 1331-1340.

[20] J.-M. Lehn, Supramolecular Chemistry: Concepts and Perspectives, VCH, New York, 1995.

[21] D. Lombardo, Langmuir 25 (2009), 3271-3275.

[22] D. Lombardo, A. Longo, R. Darcy and A. Mazzaglia, Langmuir 20 (2004), 1057-1064.

[23] F. Mallamace, R. Beneduci, P. Gambadauro, D. Lombardo and S.H. Chen, Phys. A 302 (2001), 202-219.

[24] F. Mallamace, E. Canetta, D. Lombardo, A. Mazzaglia, A. Romeo, L. Monsù Scolaro and G. Maino, Phys. A 304 (2002), $235-243$.

[25] A. Mazzaglia, N. Angelini, R. Darcy, D. Lombardo, N. Micali, M.T. Sciortino, V. Villari and L. Monsú Scolaro, Chem. Eur. J. 9 (2003), 5762-5769.

[26] A. Mazzglia, N. Angelini, D. Lombardo, N. Micali, S. Patane, V. Villari and L.M. Scolaro, J. Phys. Chem. B 109 (2005), $7258-7265$

[27] N. Micali, L. Monsu Scolaro, A. Romeo, D. Lombardo, P. Lesieur and F. Mallamace, Phys. Rev. E 58 (1998), $6229-6235$.

[28] N.W. Ockwig, O. Delgado-Friedrichs, M. O'Keeffe and O.M. Yaghi, Acc. Chem. Res. 38 (2005), 176-182.

[29] Result from the diluted sample at dendrimer concentration of $C=0.2 \mathrm{wt} \%$.

[30] H.-J. Schneider and A. Yatsimirsky, Principles and Methods in Supramolecular Chemistry, Wiley, New York, 2000.

[31] A. Stein, B.J. Melde and R.C. Schroden, Adv. Mater. 12 (2000), 1403-1419.

[32] D.A. Tomalia, H. Baker, J. Dewald, M. Hall, G. Kallos, S. Martin, J. Roeck, J. Ryder and P. Smith, Polym. J. 17 (1985), 117-132.

[33] D.A. Tomalia, A.M. Naylor and W.A. Goddard, Angew. Chem. Int. Ed. Engl. 29 (1990), 138-175.

[34] O.D. Velev, P.M. Tessier, A.M. Lenhoff and E.W. Kaler, Nature 401 (1999), 548-548.

[35] G.M. Whitesides and B. Grzybowski, Science 295 (2002), 2418-2421.

[36] Y. Yang and A.J. Heeger, Nature 372 (1994), 344-346. 


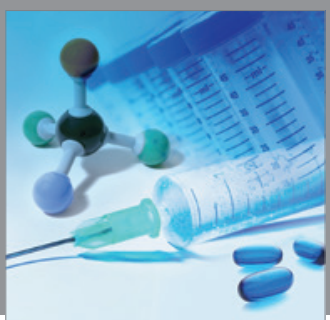

International Journal of

Medicinal Chemistry

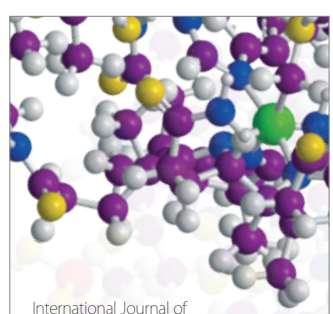

Carbohydrate Chemistry

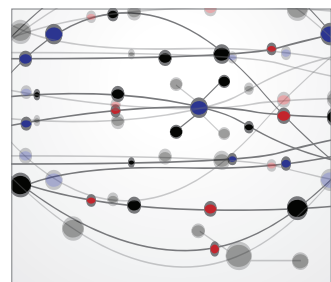

The Scientific World Journal
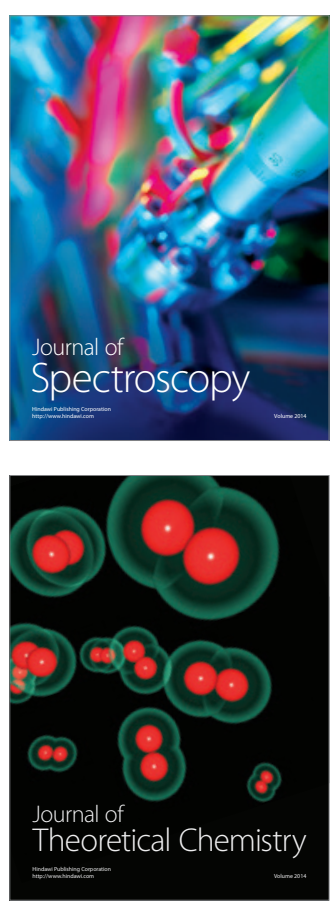
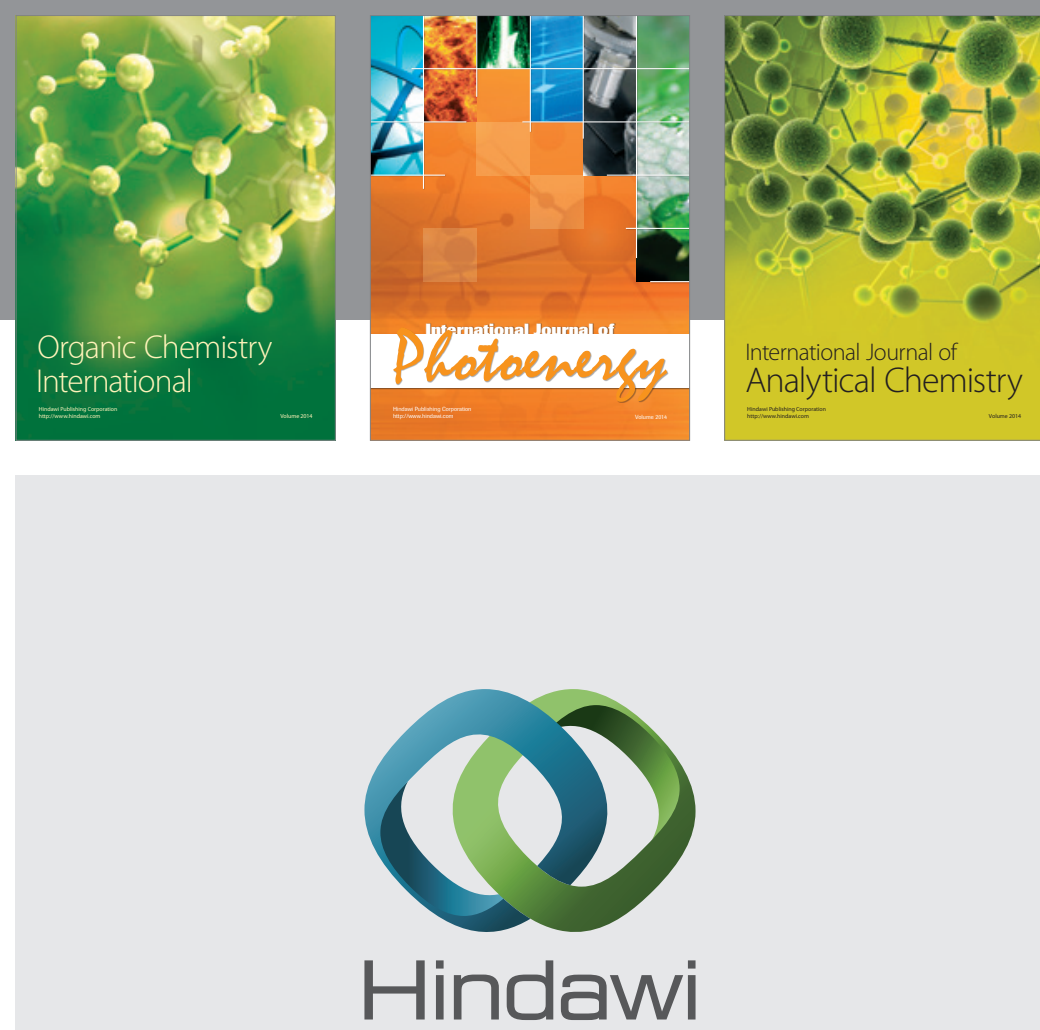

Submit your manuscripts at

http://www.hindawi.com
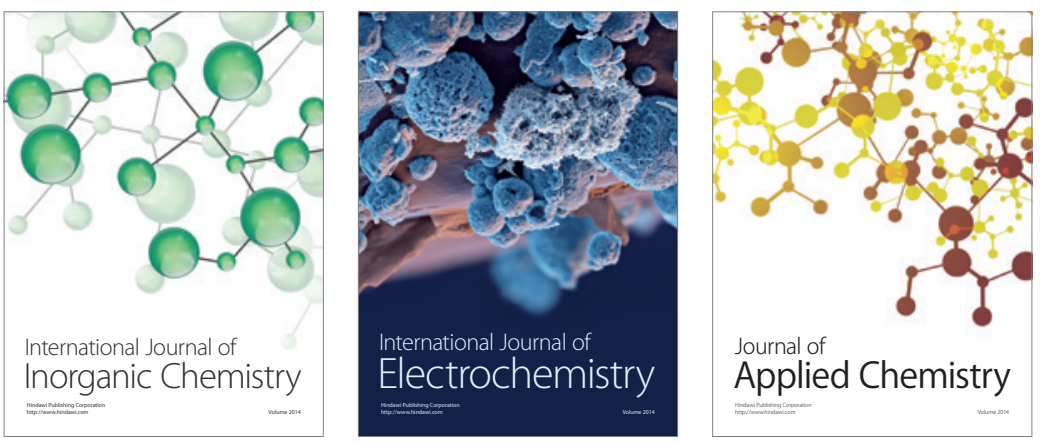

Journal of

Applied Chemistry
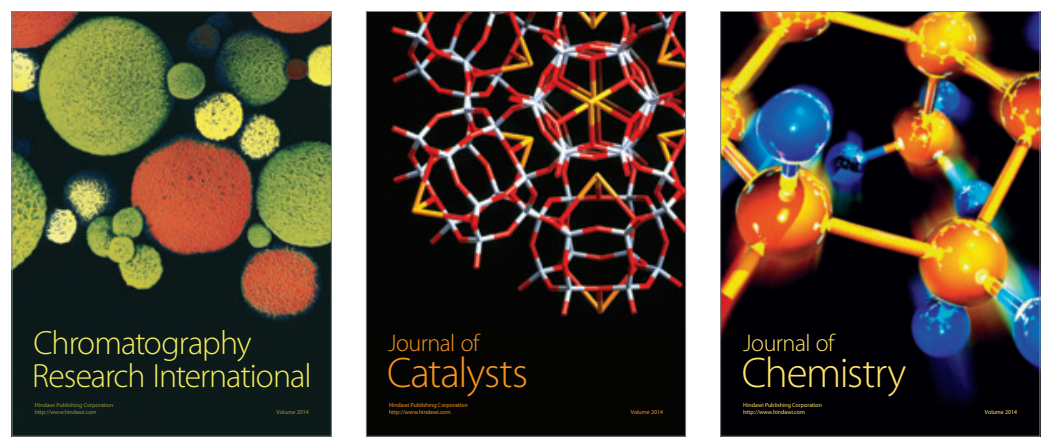
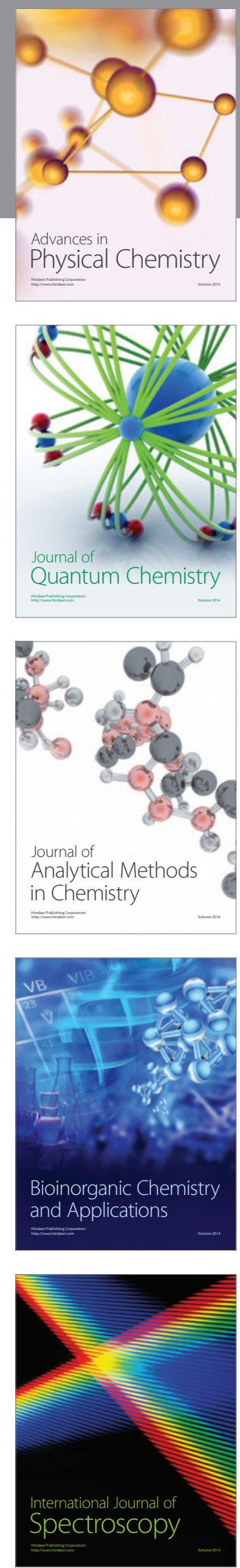